Supporting Information

for

\title{
Incorporating Ester Functionality within a Solid-State [2 + 2] Cycloaddition Reaction Based Upon Halogen Bonding Interactions
}

Samantha J. Kruse, Eric Bosch, Fayeshun Brown, and Ryan H. Groeneman

Department of Chemistry, Missouri State University, 901 South National Ave. Springfield, MO 65897, USA

Department of Biological Sciences, Webster University, 470 E. Lockwood Ave., St. Louis, MO, 63119, USA

1. Materials, General Methods and Synthesis of the Cocrystals

Page 2

2. Electrostatic Potential Calculations

Page 3

3. Single X-ray Diffraction Information and Data Tables

Page 3-5

4. ${ }^{1} \mathrm{H}$ NMR Spectroscopic Data

Page 6-9 


\section{Materials, General Methods and Synthesis of the Cocrystals}

Materials

The solvents toluene and ethanol were both purchased from Sigma-Aldrich Chemical (St. Louis, MO, USA) and used as received. The halogen bond donor 1,4-diiodoperchlorobenzene $\left(\mathrm{C}_{6} \mathrm{I}_{2} \mathrm{Cl}_{4}\right)^{1}$ as well as the ester-based reactant molecules (4-PAMe), (3-PAMe), and (2-PAMe) ${ }^{2}$ were synthesized by previously reported methods.

General Methods

All photoreactions were conducted in an ACE Glass photochemistry cabinet using UVradiation from a $450 \mathrm{~W}$ medium-pressure mercury lamp. Each cocrystal was placed between a pair of Pyrex glass plates for irradiation. The photoreactivity and the overall yield for the photoreaction were determined by using ${ }^{1} \mathrm{H}$ NMR Spectroscopy on a Bruker Avance $400 \mathrm{MHz}$ spectrometer using DMSO- $d_{6}$ as the solvent.

Synthesis of $\left(\mathrm{C}_{6} \mathrm{I}_{2} \mathrm{Cl}_{4}\right) \bullet(4-\mathrm{PAMe})$

The formation of the cocrystal $\left(\mathrm{C}_{6} \mathrm{I}_{2} \mathrm{Cl}_{4}\right) \bullet(4-\mathrm{PAMe})$ was achieved by dissolving $25 \mathrm{mg}$ of $\mathrm{C}_{6} \mathrm{I}_{2} \mathrm{Cl}_{4}$ in $2 \mathrm{~mL}$ of toluene which was then combined with $8.7 \mathrm{mg}$ of 4-PAMe in $2 \mathrm{~mL}$ of ethanol (1:1 molar equivalent). Crystals suitable for X-ray diffraction formed within two days after slow evaporation.

Synthesis of $\left(\mathrm{C}_{6} \mathrm{I}_{2} \mathrm{Cl}_{4}\right) \cdot 2(3-\mathrm{PAMe})$

In a similar manner as before, the formation of the cocrystal $\left(\mathrm{C}_{6} \mathrm{I}_{2} \mathrm{Cl}_{4}\right) \cdot 2(3-\mathrm{PAMe})$ was realized by dissolving $25 \mathrm{mg}$ of $\mathrm{C}_{6} \mathrm{I}_{2} \mathrm{Cl}_{4}$ in $2 \mathrm{~mL}$ of toluene and then combined it with $8.7 \mathrm{mg}$ of 
3-PAMe in $2 \mathrm{~mL}$ of ethanol (1:1 molar equivalent). Again, single crystals suitable for X-ray diffraction formed within two days after some evaporation of the solvents.

Synthesis of $\left(\mathrm{C}_{6} \mathrm{I}_{2} \mathrm{Cl}_{4}\right) \cdot(2-\mathrm{PAMe})$

The formation of the cocrystal $\left(\mathrm{C}_{6} \mathrm{I}_{2} \mathrm{Cl}_{4}\right) \bullet(2$-PAMe) was realized by dissolving $25 \mathrm{mg}$ of $\mathrm{C}_{6} \mathrm{I}_{2} \mathrm{Cl}_{4}$ in $2 \mathrm{~mL}$ of toluene which was then combined with $8.7 \mathrm{mg}$ of 2-PAMe in $2 \mathrm{~mL}$ of ethanol (1:1 molar equivalent). As before, single crystals suitable for X-ray diffraction formed within two days after some evaporation of the solvents.

\section{Electrostatic Potential Calculations}

The electrostatic potential energy surface for 4-PAMe, 3-PAMe, and 2-PAMe were determined using the Spartan'10 molecular modeling program ${ }^{3}$ using density functional theory (DFT) at the B3LYP/6- 311++G** level.

\section{Single Crystal X-ray Diffraction Data Collection}

A single crystal of each cocrystal was mounted on a MiTeGen cryoloop in a random orientation for X-ray data collection. A Bruker Venture Duo Photon-II single crystal X-Ray diffractometer equipped with an Oxford Cryostream device was used for all data collection. Apex II and SAINT software packages were used for data collection and integration, respectively. The data were corrected for systematic errors using SADABS ${ }^{4}$ based on the Laue symmetry using equivalent reflections. Structure solution and refinement were carried out using the SHELXTL-PLUS software package. ${ }^{5}$ All structures were solved by direct methods with a full matrix least-squares refinement where all non-hydrogen atoms were refined anisotropically to convergence. In addition, all hydrogen atoms were treated using an appropriate riding model 
(AFIX m3). X-ray diffraction and refinement data for all cocrystals and photoproducts are given in Table 1 and Table 2.

Table 1. Crystallographic diffraction and refinement data for all cocrystals.

\begin{tabular}{|c|c|c|c|}
\hline cocrystal & $\left(\mathrm{C}_{6} \mathrm{I}_{2} \mathrm{Cl}_{4}\right) \cdot(4-\mathrm{PAMe})$ & $\left(\mathrm{C}_{6} \mathrm{I}_{2} \mathrm{Cl}_{4}\right) \cdot 2(3-\mathrm{PAMe})$ & $\left(\mathrm{C}_{6} \mathrm{I}_{2} \mathrm{Cl}_{4}\right) \cdot(2-\mathrm{PAMe})$ \\
\hline chemical formula & $\mathrm{C}_{30} \mathrm{H}_{18} \mathrm{Cl}_{8} \mathrm{I}_{4} \mathrm{~N}_{2} \mathrm{O}_{4}$ & $\mathrm{C}_{24} \mathrm{H}_{18} \mathrm{Cl}_{4} \mathrm{I}_{2} \mathrm{~N}_{2} \mathrm{O}_{4}$ & $\mathrm{C}_{15} \mathrm{H}_{9} \mathrm{Cl}_{4} \mathrm{I}_{2} \mathrm{NO}_{2}$ \\
\hline crystal system & Monoclinic & Triclinic & Triclinic \\
\hline space group & $\mathrm{Cc}$ & $\mathrm{P} \overline{1}$ & $\mathrm{P}_{\overline{1}}$ \\
\hline $\mathrm{a} / \mathrm{A}$ & $4.0952(2)$ & $4.0647(5)$ & $8.2370(3)$ \\
\hline $\mathrm{b} / \AA$ & $28.2895(10)$ & $9.2406(11)$ & $9.2267(4)$ \\
\hline $\mathrm{c} / \AA \AA$ & $16.7967(6)$ & 37.473(4) & $13.1985(5)$ \\
\hline$\alpha /{ }^{\circ}$ & 90 & $87.384(4)$ & $103.098(2)$ \\
\hline$\beta /^{\circ}$ & $96.9480(10)$ & $88.721(4)$ & $90.168(2)$ \\
\hline$\gamma /{ }^{\circ}$ & 90 & $80.110(4)$ & $102.568(2)$ \\
\hline $\mathrm{V} / \mathrm{A}^{3}$ & 1931.63(14) & $1385.0(3)$ & 952.14(7) \\
\hline$\rho_{\text {calc }} / \mathrm{g} \mathrm{cm}^{-3}$ & 2.169 & 1.904 & 2.200 \\
\hline $\mathrm{T} / \mathrm{K}$ & 302 & 290 & 296 \\
\hline $\mathrm{Z}$ & 4 & 2 & 2 \\
\hline radiation type & Mo K $\alpha$ & Mo K $\alpha$ & Mo Ka \\
\hline absorption coefficient, $\mu / \mathrm{mm}^{-1}$ & 3.817 & 2.690 & 3.872 \\
\hline no. of reflections measured & 18024 & 20504 & 17092 \\
\hline no. of independent reflections & 3898 & 5633 & 6916 \\
\hline $\mathrm{R}_{\mathrm{int}}$ & 0.0528 & 0.0670 & 0.0271 \\
\hline $\mathrm{R}_{1}(\mathrm{I}>2 \sigma(\mathrm{I}))$ & 0.0293 & 0.0826 & 0.0337 \\
\hline $\mathrm{wR}\left(\mathrm{F}^{2}\right)(\mathrm{I}>2 \sigma(\mathrm{I}))$ & 0.0553 & 0.2204 & 0.0765 \\
\hline $\mathrm{R}_{1}$ (all data) & 0.0334 & 0.0930 & 0.0550 \\
\hline $\mathrm{wR}\left(\mathrm{F}^{2}\right)$ (all data) & 0.0577 & 0.2311 & 0.0867 \\
\hline Goodness-of-fit & 1.109 & 1.088 & 1.016 \\
\hline CCDC deposition number & 1967335 & 1967336 & 1967337 \\
\hline
\end{tabular}


Table 2. Crystallographic diffraction and refinement data for crystals containing the photoproducts.

\begin{tabular}{|c|c|c|}
\hline cocrystal & $\left(4,4^{\prime}-\mathrm{BPCD}\right)$ & $\left(\mathrm{C}_{6} \mathrm{I}_{2} \mathrm{Cl}_{4}\right) \cdot\left(3,3^{\prime}-\mathrm{BPCD}\right)$ \\
\hline chemical formula & $\mathrm{C}_{36} \mathrm{H}_{36} \mathrm{~N}_{4} \mathrm{O}_{8}$ & $\mathrm{C}_{24} \mathrm{H}_{18} \mathrm{Cl}_{4} \mathrm{I}_{2} \mathrm{NO}_{4}$ \\
\hline crystal system & Monoclinic & Monoclinic \\
\hline space group & $\mathrm{P} 2{ }_{1} / \mathrm{n}$ & $\mathrm{P} 2{ }_{1} / \mathrm{c}$ \\
\hline $\mathrm{a} / \AA$ & $11.2145(16)$ & $14.8816(5)$ \\
\hline $\mathrm{b} / \AA ̊ A$ & $10.2300(14)$ & $10.5023(3)$ \\
\hline $\mathrm{c} / \AA$ & $15.073(2)$ & $17.5019(6)$ \\
\hline$\alpha /^{\circ}$ & 90 & 90 \\
\hline$\beta /^{\circ}$ & $106.176(6)$ & $99.6340(10)$ \\
\hline$\gamma /{ }^{\circ}$ & 90 & 90 \\
\hline $\mathrm{V} / \AA^{3}$ & $1660.8(4)$ & 2696.81(15) \\
\hline$\rho_{\text {calc }} / \mathrm{g} \mathrm{cm}^{-3}$ & 1.305 & 1.956 \\
\hline $\mathrm{T} / \mathrm{K}$ & 100 & 100 \\
\hline $\mathrm{Z}$ & 2 & 4 \\
\hline radiation type & Mo K $\alpha$ & Mo K $\alpha$ \\
\hline absorption coefficient, $\mu / \mathrm{mm}^{-1}$ & 0.093 & 2.763 \\
\hline no. of reflections measured & 19442 & 47146 \\
\hline no. of independent reflections & 3389 & 8150 \\
\hline $\mathrm{R}_{\text {int }}$ & 0.1194 & 0.0330 \\
\hline $\mathrm{R}_{1}(\mathrm{I}>2 \sigma(\mathrm{I}))$ & 0.0624 & 0.0265 \\
\hline $\mathrm{wR}\left(\mathrm{F}^{2}\right)(\mathrm{I}>2 \sigma(\mathrm{I}))$ & 0.1100 & 0.0479 \\
\hline $\mathrm{R}_{1}$ (all data) & 0.1478 & 0.0416 \\
\hline$w R\left(F^{2}\right)$ (all data) & 0.1346 & 0.0519 \\
\hline Goodness-of-fit & 1.028 & 1.041 \\
\hline CCDC deposition number & 1967338 & 1967339 \\
\hline
\end{tabular}




\section{4. ${ }^{1} \mathrm{H}$ NMR Spectroscopic Data}

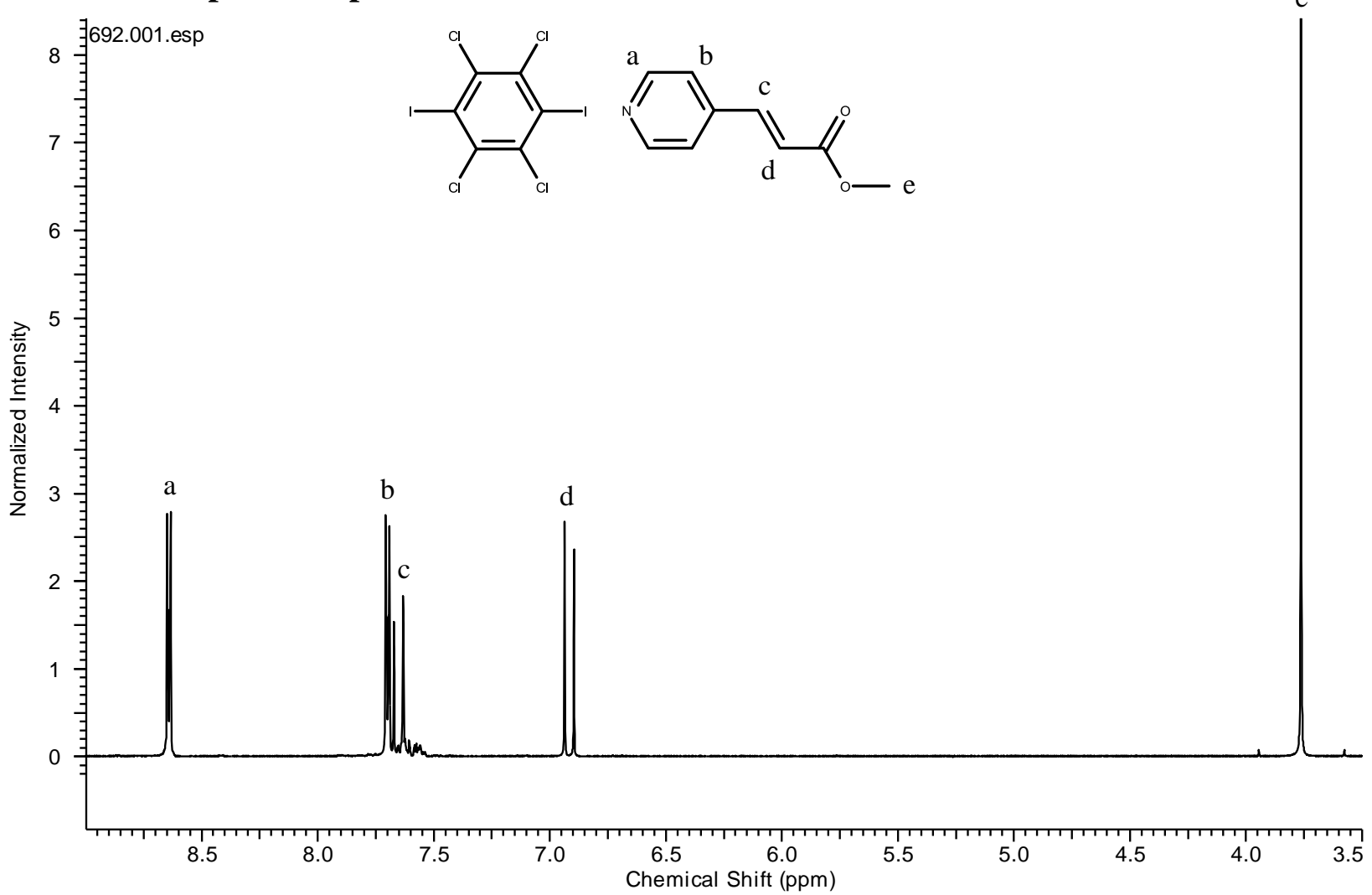

Figure S1: ${ }^{1} \mathrm{H}$ NMR spectrum of the cocrystal $\left(\mathrm{C}_{6} \mathrm{I}_{2} \mathrm{Cl}_{4}\right) \bullet(4-\mathrm{PAMe})$ before UV irradiation. (400 $\left.\mathrm{MHz}, \mathrm{DMSO}-d_{6}\right)$. 


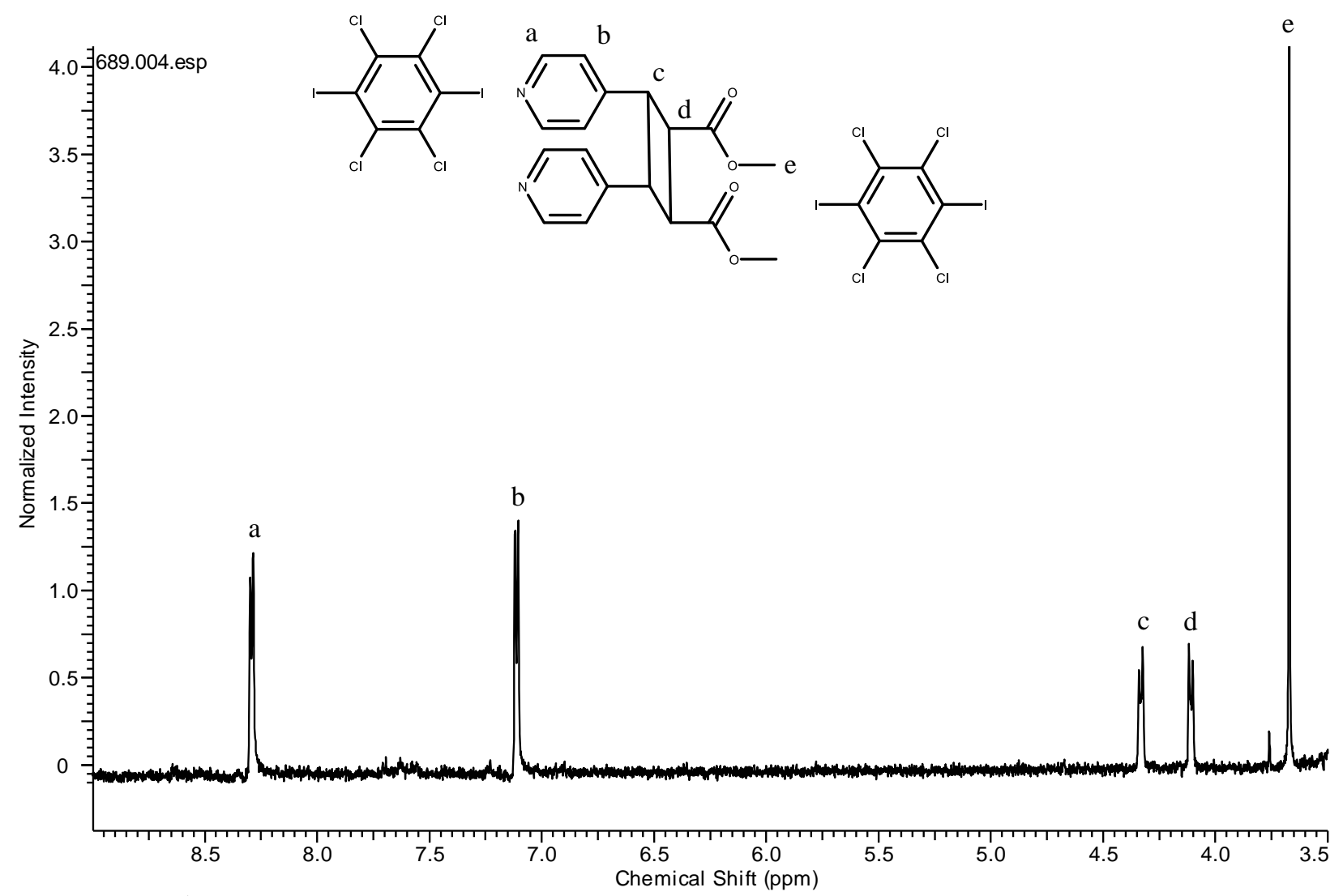

Figure S2: ${ }^{1} \mathrm{H}$ NMR spectrum of the cocrystal $\left(\mathrm{C}_{6} \mathrm{I}_{2} \mathrm{Cl}_{4}\right) \bullet(4-\mathrm{PAMe})$ after UV irradiation producing $(4,4$ '-BPCD) in a quantitative yield. (400 MHz, DMSO-d6). 


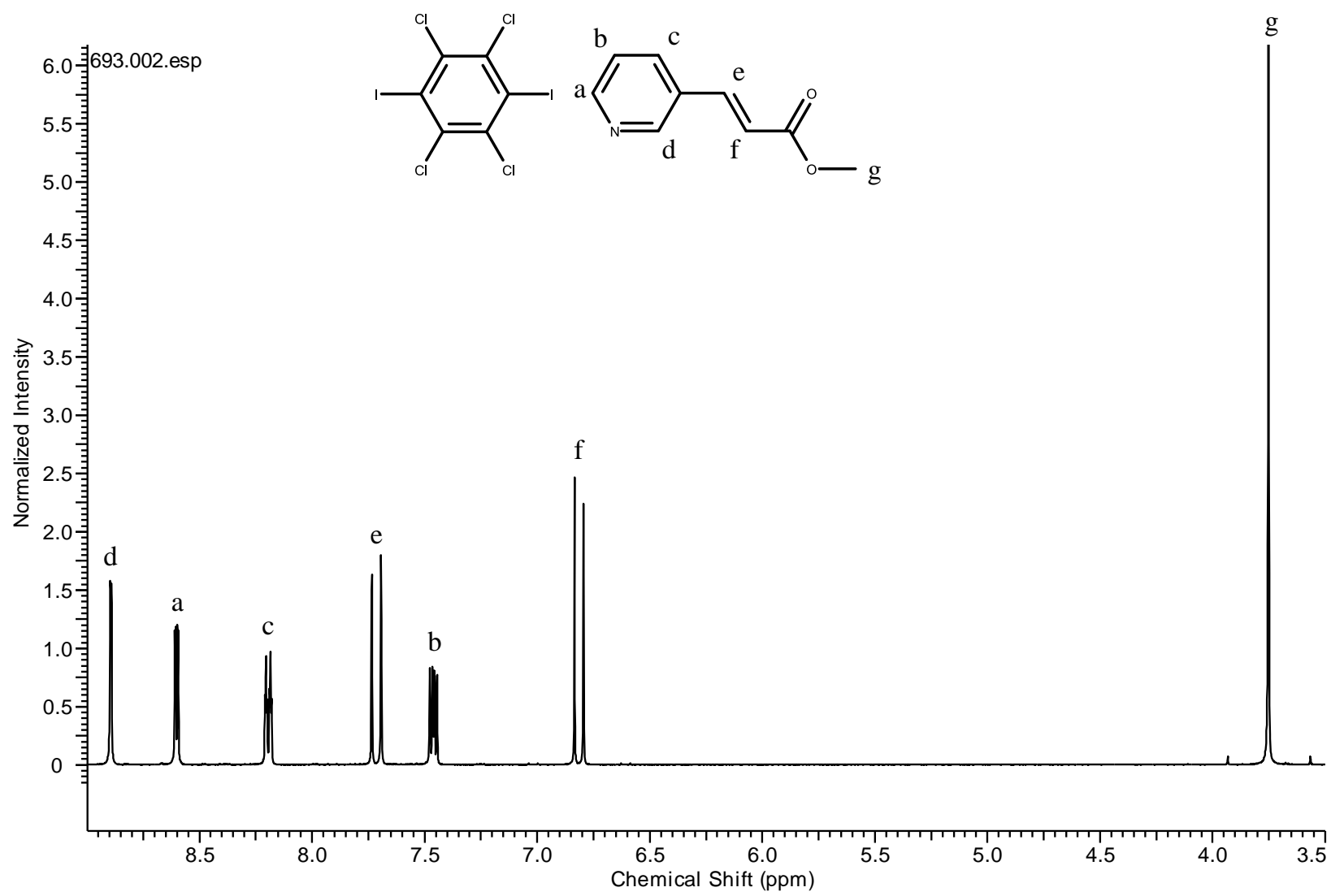

Figure S3: ${ }^{1} \mathrm{H}$ NMR spectrum of the cocrystal $\left(\mathrm{C}_{6} \mathrm{I}_{2} \mathrm{Cl}_{4}\right) \cdot 2(3$-PAMe) before UV irradiation. (400 $\left.\mathrm{MHz}, \mathrm{DMSO}-d_{6}\right)$. 


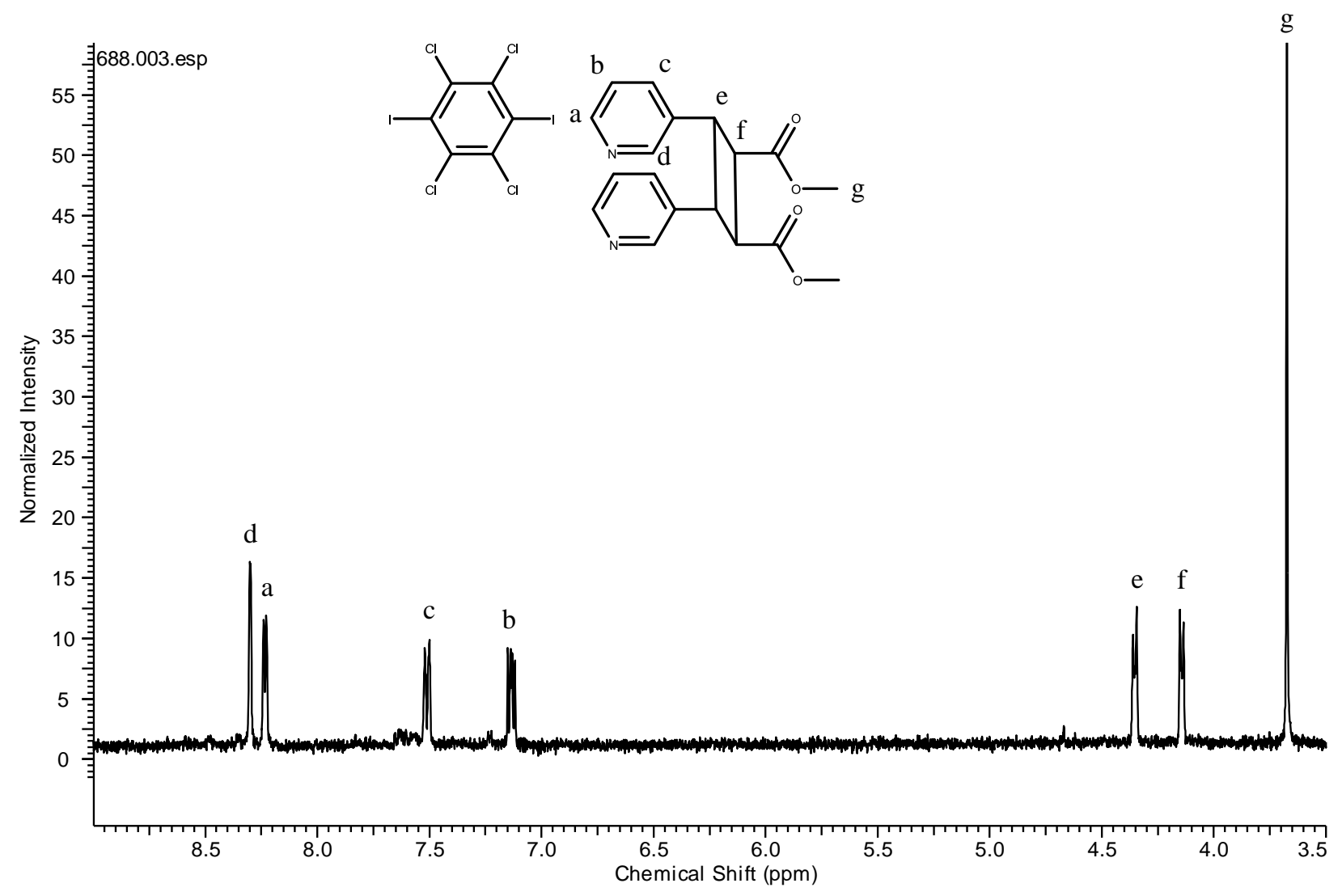

Figure S4: ${ }^{1} \mathrm{H}$ NMR spectrum of the cocrystal $\left(\mathrm{C}_{6} \mathrm{I}_{2} \mathrm{Cl}_{4}\right) \cdot 2(3-\mathrm{PAMe})$ after UV irradiation producing (3,3'-BPCD) in a quantitative yield. (400 MHz, DMSO-d6).

\section{References}

1. Reddy, C. M.; Kirchner, M. T.; Gundakaram, R. C.; Padmanabhan, K. A.; Desiraju, G. R. Chem. Eur. J. 2006, 12, 2222.

2. Agarwal, K. C.; Knaus, E. E. J. Heterocyclic Chem. 1985, 22, 65.

3. Spartan '10, Version 1.0.1, Wavefunction, Inc. Irvine, CA, USA.

4. Krause et al., (2015) SADABS v 2016/2.

5. Sheldrick, G. M. Acta Crystallogr. 2015, C71, 3. 